\title{
Hemothorax caused by the trocar tip of the rod inserter after minimally invasive transforaminal lumbar interbody fusion: case report
}

\author{
Keishi Maruo, MD, Toshiya Tachibana, MD, Shinichi Inoue, MD, Fumihiro Arizumi, MD, and \\ Shinichi Yoshiya, MD
}

Department of Orthopaedic Surgery, Hyogo College of Medicine, Nishinomiya, Hyogo, Japan

\begin{abstract}
Minimally invasive surgery (MIS) for transforaminal lumbar interbody fusion (MIS-TLIF) is widely used for lumbar degenerative diseases. In the paper the authors report a unique case of a hemothorax caused by the trocar tip of the rod inserter after MIS-TLIF. A 61-year-old woman presented with thigh pain and gait disturbance due to weakness in her lower right extremity. She was diagnosed with a lumbar disc herniation at L1-2 and the MIS-TLIF procedure was performed. Immediately after surgery, the patient's thigh pain resolved and she remained stable with normal vital signs. The next day after surgery, she developed severe anemia and her hemoglobin level decreased to $7.6 \mathrm{~g} / \mathrm{dl}$, which required blood transfusions. A chest radiograph revealed a hemothorax. A CT scan confirmed a hematoma of the left paravertebral muscle. A chest tube was placed to treat the hemothorax. After 3 days of drainage, there was no active bleeding. The patient was discharged 14 days after surgery without leg pain or any respiratory problems. This complication may have occurred due to injury of the intercostal artery by the trocar tip of the rod inserter. A hemothorax after spine surgery is a rare complication, especially in the posterior approach. The rod should be caudally inserted in the setting of the thoracolumbar spine.
\end{abstract}

http://thejns.org/doi/abs/10.3171/2015.6.SPINE15488

KEY WORDS hemothorax; complication; spine surgery; minimally invasive transforaminal lumbar interbody fusion; upper lumbar disc herniation; rod inserter

$\mathrm{T}$ RANSFORAMINAL lumbar interbody fusion (TLIF) is an effective surgical treatment for lumbar degenerative conditions. Minimally invasive surgery (MIS) techniques for TLIF (MIS-TLIF) have been demonstrated to reduce the damage of paraspinal muscles as well as decrease blood loss and postoperative pain, shorten length of hospital stay, and be more cost-effective treatments..$^{1,5,6,17,18}$ In addition, clinical outcomes including visual analog scale score and Oswestry Disability Index were clinically equivalent between MIS and open TLIF. Therefore, the MIS-TLIF technique has been widely used in the treatment of lumbar degenerative diseases. Perioperative complications in MIS-TLIF are uncommon. Several studies have suggested trends toward lower rates of surgical complications including dural tear, infection, graft malposition, neurological deficit or nerve injury, and hematoma in MIS cohorts. ${ }^{5}$ On the other hand, MIS techniques have a surgi- cal learning curve and there are some risks of MIS-specific complications such as screw malposition, vascular injury for guidewire-assisted pedicle screw insertion, and a disconnecting rod and screw system. ${ }^{9,12}$ The occurrence of a hemothorax after posterior spine surgery is a rare complication. In addition, there have been no published reports of a hemothorax after MIS-TLIF. In this paper we present a patient who experienced a hemothorax caused by the rod trocar tip after MIS-TLIF for the treatment of upper lumbar disc herniation.

\section{Case Report}

History and Examination

A 61-year-old woman presented with right thigh pain and gait disturbance along with weakness of her lower right extremity. She was diagnosed with lumbar disc her-

ABBREVIATIONS MIS = minimally invasive surgery; TLIF = transforaminal lumbar interbody fusion . SUBMITTED April 26, 2015. ACCEPTED June 11, 2015.

INCLUDE WHEN CITING Published online November 20, 2015; DOI: 10.3171/2015.6.SPINE15488. 
niation at L1-2 on MRI (Fig. 1). The patient was treated with pain management using medication and an epidural block at the local hospital; however, symptoms of intermittent claudication progressed due to right thigh pain. Surgical treatment was considered due to her persistent severe leg pain. MIS-TLIF was chosen because the disc herniation was located at the L1-2 level and it avoids significant retraction of the cauda equina and conus medullaris.

\section{Surgical Procedure}

A 3-cm longitudinal incision at the right-side L1-2 level was made for placement of the Quadrant retractor system (Medtronic Sofamor Danek). After the right-side L1-2 facet joint was removed using an osteotome and rongeurs, a discectomy was performed. Autologous bone grafts obtained from the removed facet and lamina were packed in the anterior disc space. A single polyetheretherketone cage (Capstone; Medtronic Sofamor Danek) was placed obliquely into the L1-2 disc space and then the Quadrant tube retractor system was removed. Percutaneous pedicle screws were placed through a guidewire under fluoroscopic guidance using the Sextant system (Medtronic Sofamor Danek). After pedicle screw insertion, the extenders were connected and the rod inserter and trocar tip were attached to the 2 screw-extender assemblies. A 1-cm longitudinal incision was made to path to gain access to the fascia and muscle. The rod trocar was inserted cranially until it touched the proximal screw. Positioning of the screws and rod was confirmed with anteroposterior and lateral fluoroscopy.

\section{Postoperative Course}

Immediately after surgery, the patient's thigh pain resolved and the patient remained stable with normal vitals. The next day after surgery, she developed severe anemia and her hemoglobin level decreased to $7.6 \mathrm{~g} / \mathrm{dl}$, which required blood transfusions. A chest radiograph and CT scan revealed a hemothorax (Fig. 2A and B). A plain CT scan revealed swelling of the left-side paraver-

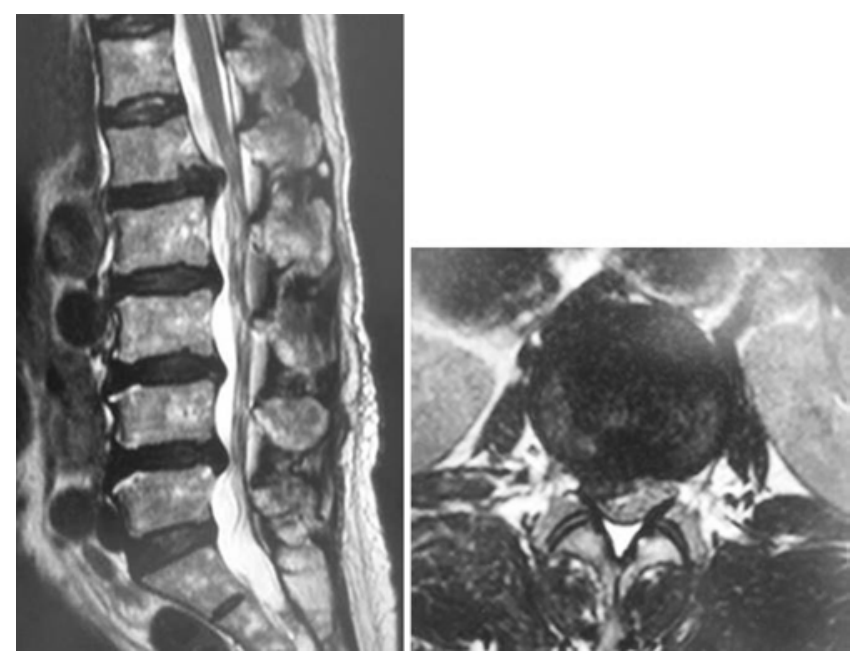

FIG. 1. Sagittal (left) and axial (right) T2-weighted MR images at L1-2 demonstrate the significantly herniated disc (left) and right-side compression of the spinal canal (right).
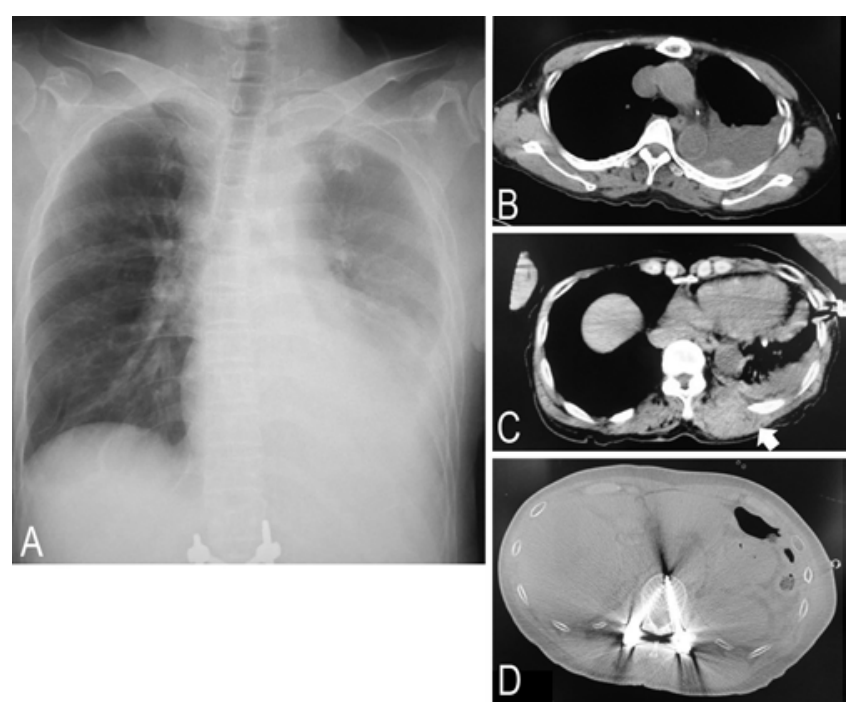

FIG. 2. Anteroposterior postoperative plain radiograph (A) and axial CT scan (B) show a hemothorax on the left side. An axial CT scan at T-11 demonstrates a hematoma (arrow) of the paravertebral muscle (C). An axial CT scan shows the location of pedicle screws at L-1 (D).

tebral muscle at the T-11 level (Fig. 2C). We evaluated the perforation of the aorta branches during pedicle screw insertion. The pedicle screws did not breach the lateral and anterior vertebral wall. In addition, screw tips at the L-1 and L-2 level were located in the retroperitoneal space that were not in the thoracic cavity (Fig. 2D). However, the enhanced CT images did not confirm arterial bleeding. A chest tube was placed between the fifth and sixth ribs to treat the hemothorax in the left thorax. Initially, a total of $300 \mathrm{ml}$ of bloody drainage was confirmed but active bleeding had already stopped. Her blood hemoglobin level did not decrease after blood transfusions. The chest tube was removed after 3 days of drainage. The patient was discharged 14 days after surgery without leg pain or any respiratory problems. This complication may have occurred due to injury of the intercostal artery by the trocar tip of the rod inserter.

\section{Discussion}

The complication rate of MIS-TLIF is $0-40 \% .^{5,9,12,20,22}$ Surgical-procedure related complications after MIS-TLIF include dural tear, infection, graft malposition, screw malposition, neurological deficit or nerve injury, and hematoma. Wang and Zhou reported that the overall incidence of perioperative complications associated with MIS-TLIF surgery was $37 \%$ in 204 patients. $^{20}$ The rate of surgical procedure-related complications and medical complications were $23 \%$ and $14 \%$, respectively. Leg sensory disturbance was the most common complication, occurring in $12 \%$; the second most common complication was dural tear, occurring in $10 \%$. Several studies reported trends toward lower complications rates in the MIS group than the open group..$^{20} \mathrm{On}$ the other hand, Lau et al. noted that the MIS-TLIF group had a higher rate of complications, which might have been associated with the learning curve. ${ }^{9}$ Wang et al. reported that MIS-TLIF is a safe and 


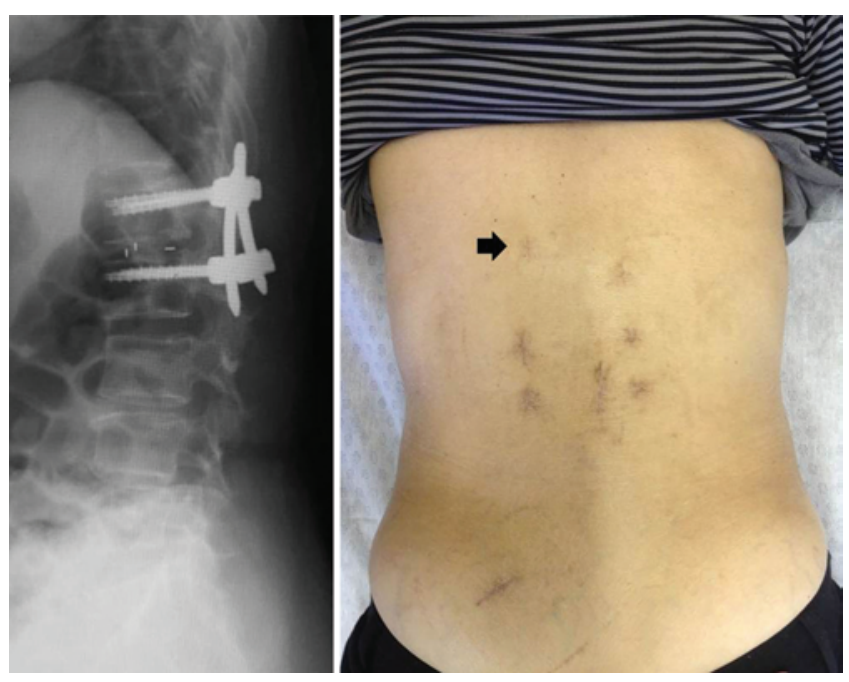

FIG. 3. A lateral plain radiograph (left) shows the proximal screw head is deeper than the distal screw head. The skin incision on the left side (right) is located more cranially than the right side (arrow). Figure is available in color online only.

effective procedure for disc herniation in the thoracolumbar junction. ${ }^{21}$ There were no major complications after MIS-TLIF for treatment of upper lumbar disc herniation.

A hemothorax is a collection of blood in the pleural space and may be caused by penetrating trauma. A hemothorax after spine surgery is a rare complication, especially in the posterior approach. Most previously reported hemothorax cases were related to the anterior approach, caused by anterior instrumentations or thoracoplasty. ${ }^{2,4,7,8,11,14,15,19}$ Only 5 articles have been published involving a hemothorax after spine surgery via the posterior approach. ${ }^{3,10,13,16}$ Modi et al. reported 3 cases of hemothoraxes after posterior pedicle screw instrumentation surgery in patients with flaccid neuromuscular scoliosis. They concluded that the cause of the hemothorax was breaching of the lateral wall of the pedicle or the anterior vertebral body cortex during
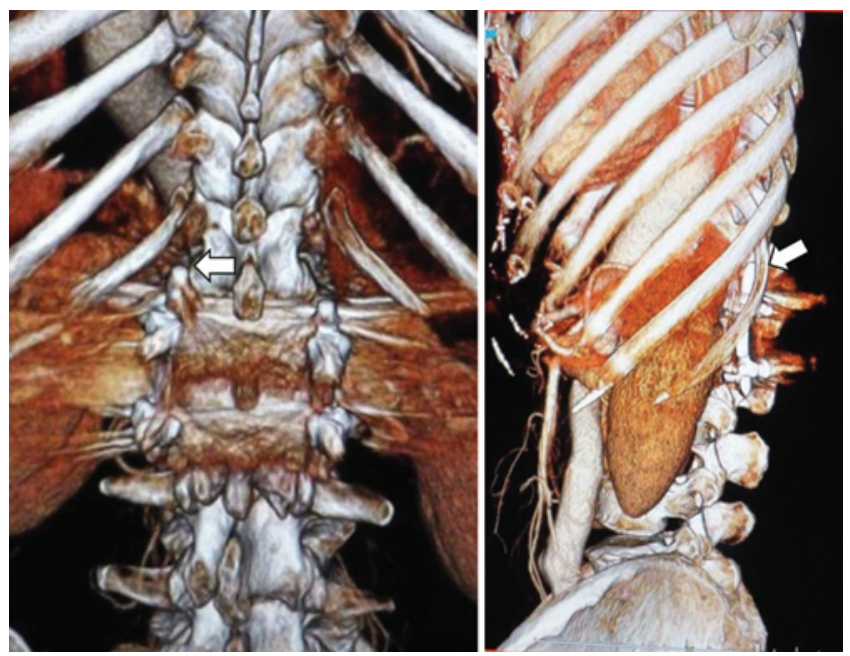

FIG. 4. Three-dimensional CT scans show the apex of the left-side rod is located close to the bottom of the twelfth rib (arrow). Figure is available in color online only.

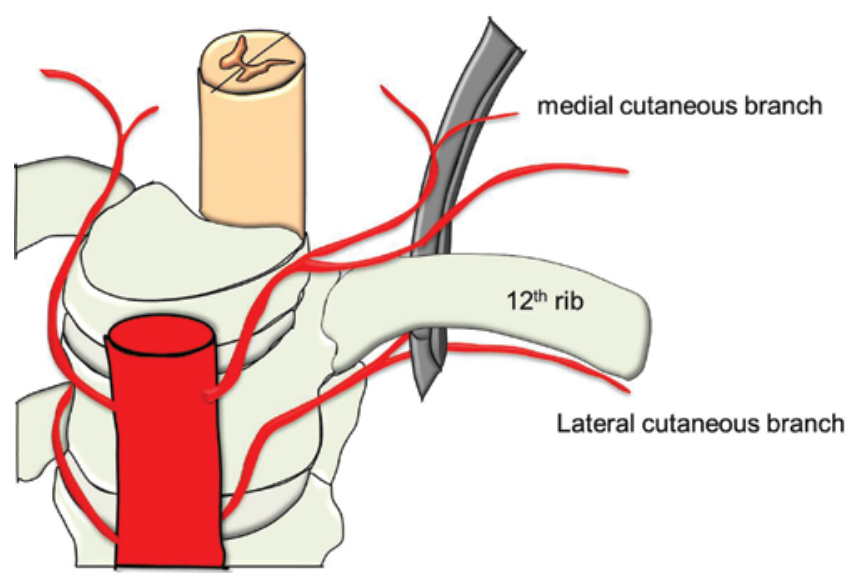

FIG. 5. The lateral cutaneous branch of the intercostal artery was injured by the rod trocar tip during its path through the fascia and muscle. Copyright Keishi Maruo. Published with permission. Figure is available in color online only.

pedicle screw probing or insertion..$^{10}$ Pang et al. reported a hemothorax caused by a Gelpi retractor during posterior surgery for adolescent idiopathic scoliosis. ${ }^{14}$

In the present case, a postoperative CT scan confirmed a hematoma of the left paravertebral muscle at the T-11 level, which is the skin entry point of the trocar tip. The trocar tip of the rod inserter was inserted cranially through the left paravertebral muscle. The radiograph confirmed the proximal screw head position was deeper than the distal screw head (Fig. 3 left). Therefore, the left skin incision of the rod insertion was located more cranially than the right side (Fig. 3 right). This means the rod trajectory was started at a deeper level through the twelfth rib. A 3D CT scan revealed there is some possibility that the rod trocar tip passed by the twelfth intercostal artery (Fig. 4). Therefore, the lateral cutaneous branch of the intercostal artery was injured by the rod trocar tip during its path through the fascia and muscle (Fig. 5). To our knowledge, there have been no published reports of a hemothorax after MIS-TLIF caused by rod trocar tip. The rod should be inserted caudally in the setting of thoracolumbar levels.

\section{References}

1. Archavlis E, Carvi y Nievas M: Comparison of minimally invasive fusion and instrumentation versus open surgery for severe stenotic spondylolisthesis with high-grade facet joint osteoarthritis. Eur Spine J 22:1731-1740, 2013

2. Baaj AA, Dakwar E, Le TV, Smith DA, Ramos E, Smith WD, et al: Complications of the mini-open anterolateral approach to the thoracolumbar spine. J Clin Neurosci 19:1265-1267, 2012

3. Barcelos AC, Botelho RV: Treatment of subacute thoracic spine fracture-dislocation by total vertebrectomy and spine shortening: technical note. J Neurosurg Spine 18:194-200, 2013

4. Geervliet PC, van Royen BJ, Vonk Noordegraaf A, Kranendonk SE, David EF, Paul MA: Late spontaneous hemothorax complicating anterior spinal instrumentation in adolescent idiopathic scoliosis. Spine (Phila Pa 1976) 32:E730-E733, 2007

5. Goldstein CL, Macwan K, Sundararajan K, Rampersaud YR: Comparative outcomes of minimally invasive surgery for 
posterior lumbar fusion: a systematic review. Clin Orthop Relat Res 472:1727-1737, 2014

6. Habib A, Smith ZA, Lawton CD, Fessler RG: Minimally invasive transforaminal lumbar interbody fusion: a perspective on current evidence and clinical knowledge. Minim Invasive Surg 2012:657342, 2012

7. Hsieh PH, Chen WJ, Chen LH, Niu CC: An unusual complication of anterior spinal instrumentation: hemothorax contralateral to the side of the incision. A case report. J Bone Joint Surg Am 81:998-1001, 1999

8. Konno S, Yabuki S, Kinoshita T, Kikuchi S: Combined laminectomy and thoracoscopic resection of dumbbell-type thoracic cord tumor. Spine (Phila Pa 1976) 26:E130-E134, 2001

9. Lau D, Lee JG, Han SJ, Lu DC, Chou D: Complications and perioperative factors associated with learning the technique of minimally invasive transforaminal lumbar interbody fusion (TLIF). J Clin Neurosci 18:624-627, 2011

10. Modi HN, Suh SW, Hong JY, Cho JW, Park JH, Yang JH: Treatment and complications in flaccid neuromuscular scoliosis (Duchenne muscular dystrophy and spinal muscular atrophy) with posterior-only pedicle screw instrumentation. Eur Spine J 19:384-393, 2010

11. Modi HN, Suh SW, Hong JY, Yang JH: Posterior multilevel vertebral osteotomy for severe and rigid idiopathic and nonidiopathic kyphoscoliosis: a further experience with minimum two-year follow-up. Spine (Phila Pa 1976) 36:11461153,2011

12. Nandyala SV, Fineberg SJ, Pelton M, Singh K: Minimally invasive transforaminal lumbar interbody fusion: one surgeon's learning curve. Spine J 14:1460-1465, 2014

13. Neves RP, Oliveira VC, Costa LM, Soares DF, Cardoso $\mathrm{PF}$, Costa PG, et al: Major complications following total en bloc spondylectomy for giant-cell tumor. J Surg Case Rep 2014:rjt131, 2014

14. Pang L, Watanabe K, Toyama Y, Matsumoto M: Massive hemothorax caused by Gelpi retractor during posterior correction surgery for adolescent idiopathic scoliosis: a case report. Scoliosis 9:17, 2014

15. Payer M, Sottas C: Mini-open anterior approach for corpectomy in the thoracolumbar spine. Surg Neurol 69:25-32, 2008

16. Salas N, Prébet R, Guenoun B, Gayet LE, Pries P: Vertebral body cage use in thoracolumbar fractures: outcomes in a prospective series of 23 cases at 2 years' follow-up. Orthop Traumatol Surg Res 97:602-607, 2011
17. Seng C, Siddiqui MA, Wong KP, Zhang K, Yeo W, Tan SB, et al: Five-year outcomes of minimally invasive versus open transforaminal lumbar interbody fusion: a matched-pair comparison study. Spine (Phila Pa 1976) 38:2049-2055, 2013

18. Sulaiman WA, Singh M: Minimally invasive versus open transforaminal lumbar interbody fusion for degenerative spondylolisthesis grades 1-2: patient-reported clinical outcomes and cost-utility analysis. Ochsner J 14:32-37, 2014

19. Verheyden AP, Hoelzl A, Lill H, Katscher S, Glasmacher S, Josten C: The endoscopically assisted simultaneous posteroanterior reconstruction of the thoracolumbar spine in prone position. Spine J 4:540-549, 2004

20. Wang J, Zhou Y: Perioperative complications related to minimally invasive transforaminal lumbar fusion: evaluation of 204 operations on lumbar instability at single center. Spine J 14:2078-2084, 2014

21. Wang J, Zhou Y, Zhang ZF, Li CQ, Zheng WJ, Huang B: Disc herniation in the thoracolumbar junction treated by minimally invasive transforaminal interbody fusion surgery. J Clin Neurosci 21:431-435, 2014

22. Wu WJ, Liang Y, Zhang XK, Cao P, Zheng T: Complications and clinical outcomes of minimally invasive transforaminal lumbar interbody fusion for the treatment of one- or two-level degenerative disc diseases of the lumbar spine in patients older than 65 years. Chin Med J (Engl) 125:2505-2510, 2012

\section{Disclosures}

The authors report no conflict of interest concerning the materials or methods used in this study or the findings specified in this paper.

\section{Author Contributions}

Conception and design: Maruo. Acquisition of data: Maruo. Analysis and interpretation of data: Maruo. Drafting the article: Maruo. Critically revising the article: Maruo. Reviewed submitted version of manuscript: all authors.

\section{Correspondence}

Keishi Maruo, Department of Orthopaedic Surgery, Hyogo College of Medicine, Mukogawacho 1-1, Nishinomiya, Hyogo 6638501, Japan.email: kmaruo@hyo-med.ac.jp. 